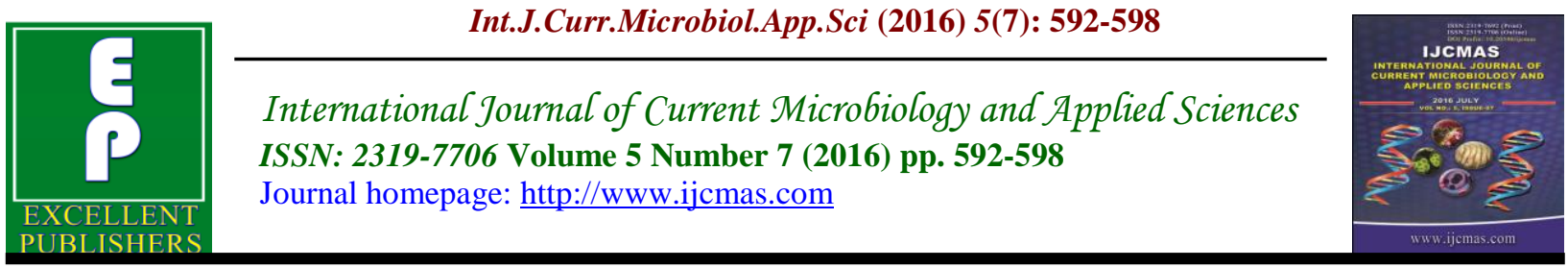

Original Research Article

http://dx.doi.org/10.20546/ijcmas.2016.507.066

\title{
Prevalence of Human Leucocyte Antigen B17 in Spondyloarthritis Patients in a Tertiary Care Center in South India Detected Microlymphocytotoxicity Test
}

\author{
Therese Mary Dhason ${ }^{1 *}$, Euphrasia Latha Jairaj ${ }^{2}$ and Rajeswari Sankaralingam ${ }^{3}$ \\ ${ }^{1}$ Senior Assistant Professor of Microbiology (Immunology), Institute of Rheumatology, \\ Madras Medical College, Chennai, India \\ ${ }^{2}$ Professor of Microbiology (Immunology), Instiute of Rheumatology, \\ Madras Medical College, Chennai, India \\ ${ }^{3}$ Director and Head, Institute of Rheumatology, Madras Medical College, Chennai, India \\ *Corresponding author
}

\section{Keywords}

Spondyloarthritis,

HLA phenotyping,

Microlympho-

cytotoxicity test, HLA-B17.

\begin{tabular}{l}
\hline Article Info \\
\hline Accepted: \\
20 June 2016 \\
Available Online: \\
10 July 2016
\end{tabular}

A B S T R A C T

To detect the prevalence of Human leukocyte antigen (HLA)B17 in Spondyloarthritis (SpA) patients from South India at a Tertiary Care Center by Microlymphocytotoxicity test, a serological method. A case control study was undertaken at a Tertiary Care Center in Chennai to find out the prevalence of HLAB17 in patients with clinical features of Spondyloarthritis. Blood samples were collected from 75 Spondyloarthritis patients and 85 healthy controls. HLA Class I phenotyping was done by microlymphocytotoxicity test using HLA Terasaki tissue typing trays, procured from One Lambda, California (CA), United States of America (USA). HLA class IB antigen distribution in Spondyloarthritis was B27 in 20(26.66\%)patients, B17 in 19(25.33\%) and B51 in10(13.33\%). The other class IB antigens were detected in low frequency. HLA-A1 was detected in 15(78.94) of the B17 positive Spondyloarthritis patients. Among the controls HLA class IB antigen distribution was B7in12(14.11\%)patients B17, B13, B40, B35, in 8 each (9.4\%). HLA-A1 was positive in $2(25 \%)$ of the B17 positive controls. Prevalence of HLA$\mathrm{B} 17$ in Spondyloarthritis patients $(\mathrm{p}=0.0104)$ and prevalence of HLA-A1 $(\mathrm{p}=0.0248)$ in $\mathrm{B} 17$ positive patients were statistically significant as compared to controls. HLA-B27 was the first common antigen and HLA-B17 was the second common HLA class 1B antigen detected in our SpA patients. HLA-A1 had an increased prevalence in our patients. Hence we conclude that HLA-B17 and HLAA1 have a possible role in Spondyloarthritis in our population. The role of these antigens in the pathogenesis of SpA has to be elucidated by large clinical trials.

\section{Introduction}

Spondyloarthritis is a group of inflammatory disorders with overlapping clinical manifestations and shared genetic markers. Ankylosing spondylitis (AS), reactive arthritis (ReA), undifferentiated Spondylo- arthritis and Psoriatic arthritis (PsA) and arthritis associated with inflammatory bowel disease(IBD) constitute SpA. Patients present with either predominantly axial or peripheral or as an overlap between these 
two patterns and with a varying association with HLA-B27. Genetics play a major role in the etiology of ankylosing spondylitis. The gene with the greatest contribution to ankylosing spondylitis is B27. It has been estimated that about $90 \%$ of the pathogenesis ofAS is genetically determined and that the contribution of HLA-B27 is about $50 \%$ of the genetic risk, making HLA-B-27 by far the most important risk factor (Brown et al., 1997; Kaipiainen-Seppanen et al., 1997). HLAB27 is an allele of the HLA-B locus of the human leukocyte antigen (HLA) class 1 antigen. Multiple subtypes of HLA-B27 have been defined (Kaipiainen-Seppanen et al., 1997).

Population and family studies have shown that HLA-B60 increases susceptibility to AS (Gary). Psoriatic arthritis is associated with defined HLA association -B27, B17, Cw6, DR4, DR7 and others. HLA-B27 has a special propensity to misfold in the endoplasmic reticulum and thereby to promote an unfolded protein response, which in turn modulates the functional behaviour and cytokine production of myeloid cells. Many genes can cause Spondyloarthritis. Up to 30 genes have been found.

The main symptoms are low back pain, pain and swelling in the arms and legs. Ankylosing Spondylitis (AS) tends to start in the teens and 20s with males being affected two to three times more than female. Extra articular manifestations like uveitis, gut inflammation, aortitis, aortic regurgitation, glomerulonephritis, and pulmonary manifestation can be present. A definite diagnosis of AS is usually established by radiographic evidence of bilateral sacroilitis. In most adult patients, AS can be diagnosed clinically without the HLA-B27 test. This assessment has no additional value in established disease or as a pure screening tool (Khan et al., 1982). However in young patients with inflammatory chronic back pain, a positive HLA-B27 test increases the likelihood of having AS particularly if imaging of the sacroiliac joints does not provide conclusive results. A normal erythrocyte sedimentation rate (ESR) or normal $\mathrm{C}$-reactive protein level does not exclude active disease. An elevated ESR or CRP is reported in up to $75 \%$ of patients, but it may not correlate with clinical disease activity (Khan et al., 1984). HLA phenotyping is done by the cell based method Microlymphocytotoxicity test using Terasaki HLA tissue typing trays and by molecular methods. In Microlymphocytotoxicity test viable lymphocytes are incubated with complement binding HLA ClassI antibodies coated in Terasaki Tissue Typing Trays. If the lymphocytes express an antigen recognized by a specific antibody, the Fab portion of the antibody binds to the antigen forming antigen-antibody complex. On addition of rabbit complement, c1q binds to the antigenantibody complex and initiates the complement cascade that leads to cell lysis. When lysed cells are more than $20 \%$ it denotes positive reaction and less than $20 \%$ denotes negative reaction (Terasaki et al., 1964).

Management of the patients is based on ASAS/EULAR recommendations and physiotherapy (Braun et al., 2010). Since SpA has a strong association with HLA class $1 \mathrm{~B}$ antigens, a study was undertaken to find out the prevalence class $1 \mathrm{~B}$ antigen apart from HLA-B27.

The study was conducted in the Institute of Rheumatology at Madras Medical College, Chennai a Tertiary Care Centre. It was a case control study with clinical features of SpA with 75 patients and 85 age and sex matched controls. 


\section{Materials and Methods}

HLA class 1 (A, B, C) phenotyping was done by microlymphocytotoxity test using Terasaki HLA class 1 Tissue Typing Trays (72 wells) coated with HLA Class I specific monoclonal antibodies procured from One Lambda,CA(fig 1).

$5 \mathrm{ml}$ of blood sample was collected in a test tube containing $50 \mu 1$ of heparin. Heparinised blood was diluted with equal volume of phosphate buffered saline (PBS). Peripheral blood lymphocytes were separated from whole blood by density gradient separation. For this a lymphocyte separation medium which contains Ficoll-Paque Plus is used. Bøyum noted that the low viscosity of Ficoll, ${ }^{\mathrm{TM}}$ compared to the other polymeric erythrocyte aggregating agents, makes it possible to devise a lymphocyte isolation procedure involving a short, low speed centrifugation (Boyum et al., 1964). A solution of Ficoll and sodium metrizoate of the proper density and osmotic strength was placed in a centrifuge tube. Blood was layered on top, and the two-phase system was centrifuged at a low speed for a short time. The erythrocytes and granulocytes sediment to the bottom of the tube, and the purified lymphocytes could be collected from the interface between the two phases (fig 2). In a conical graduated centrifuge tube $3 \mathrm{ml}$ of lymphocyte separation medium(LSM) was taken and $3 \mathrm{ml}$ of diluted blood was layered over the LSM slowly through the sides of the tube and centrifuged at 2000 revolutions per minute for 30minutes. The buffy coat was separated and added to a test tube containing $2 \mathrm{ml}$ of PBS. To this $4 \mathrm{ml}$ of PBS was added and centrifuged at 1000 revolutions per minute for 10minutes. The supernatant was discarded, $4 \mathrm{ml}$ of PBS was added to the sediment and centrifuged at 1000 revolutions per minute for 5 minutes. The supernatant was discarded and $100 \mu \mathrm{l}$ of PBS was added to the sediment. One drop of this was placed in a Neubauer White Blood Cell (WBC) counting chamber to optimise the lymphocyte cell number required for HLA typing (fig 3). When the viable cell count in a small square was $20-40$, it was taken as optimum number of cells.

$1 \mu 1$ of viable lymphocyte was added to all the 72 wells in Terasaki Tissue Typing Trays and incubated at room temperature for 30 minutes. After 30 minutes $5 \mu l$ of rabbit complement was added and incubated for 1 hour at room temperature. Then $5 \mu 1$ of $4 \%$ eosin was added and incubated for 10 minutes. $5 \mu 1$ of formal saline was added as a fixative and interpretation was done after 15 minutes using an inverted phase contrast microscope. HLA phenotype was assigned based on the percentage of lysis of cells in each well and by American Society of Histocompatibility and Immunogenetics (ASHI) scoring. Cell lysis of more than 80\% was taken as positive control (fig. 4). Presence of viable cells of more than $80 \%$ was taken as negative control (fig. 5). The phenotype frequencies for HLA Class I and Class II will vary among different populations. (Caucasians, Americans, Blacks, Orientals etc.,)

Statistics was done using SPSS software Student ' $\mathrm{t}$ ' test.

\section{Results and Discussion}

HLA - B27 was detected as the commonest class I B antigen followed by HLA-B17 (fig. 6), Other HLA B antigens positivity was as seen in (fig 7). B17 positivity was more in the males compared to females (Fig 8).

HLA - B7 was the commonest B antigen detected in healthy controls 12 (14.11\%). HLA-B13, B17, B40 and B35 had an equal distribution of $8(9.4 \%)$ each. The other B antigens were distributed in low frequency 
(table 1). HLA-B17 positivity in SpA patients was compared to HLA-B17 positivity in healthy controls.

When compared to controls HLA-B17 positivity in the diseased was statistically significant (table 2)

The other common HLA Class I Antigen detected in B17 positive SpA patients was A1. HLA-A1 was compared with controls and results are given in table 3 .

The discovery in 1973 of the strong association of human leukocyte antigen B27 (HLA-B27) with Ankylosing spondylitis and other Spondyloarthritis (SpA) strongly supported the similar genetic background. The prevalence of AS closely parallels the frequency of HLA-B27.

Axial SpA is a disease which starts normally in the third decade of life, rarely $(<5 \%)$ at an age older than 45 years, but in $10-20 \%$ of patients even between the ages of 10 and 20 years. $^{[11]}$ Thus mostly young people are affected. Interestingly, HLA-B27-positive patients have the first symptoms about 10 years earlier in comparison to HLA-B27negative patients (Rudwaleit et al., 2009).
The prevalence of axial SpA correlates directly with the prevalence of HLA - B27 (Van den Berg et al., 2012; Weber et al., 2010) in a given population and has been estimated to be between $0.1 \%$ and $1.4 \%$ in different parts of the world.

HLA-B27 is the first described a major genetic risk factor for AS but, despite the near pervasive nature of this association it has been estimated that B27 contributes only $16 \%$ of the total genetic risk. HLA-B27 is more common in Northern Europeans and is rare in Africans and Australian Aboriginals. The prevalence of AS generally follows the frequency of HLA-B27 in that population.

Our study showed an increase in the percentage of B-27 (26.66\%) in our population. It shows the ethnic variation in the distribution of HLA-B27.

The male female ratio is estimated to be $2: 1$, in early cases without radiographic visible chronic changes approaching even 1:1, indicating that women-for unclear reasonsseem to develop chronic changes later and less often (Braun et al., 2007). In our study the male female ratio of B27 was 4:1 which is slightly higher than the earlier studies.

Table.1 HLA Class 1 B Antigen Distribution in controls $(n=85)$.

\begin{tabular}{|c|c|c|c|}
\hline SI No & HLA Type & Number & Percentage \\
\hline 1 & B7 & 12 & $14.11 \%$ \\
\hline 2 & B17 & 8 & $9.4 \%$ \\
\hline 3 & B13 & 8 & $9.4 \%$ \\
\hline 4 & B40 & 8 & $9.4 \%$ \\
\hline 5 & B35 & 8 & $9.4 \%$ \\
\hline 6 & Others & 41 & $48.23 \%$ \\
\hline
\end{tabular}

Table.2 HLA B17 positivity among controls and diseased

\begin{tabular}{|c|c|c|c|}
\hline Study Group & B17 Positive & B17 Negative & Total \\
\hline Control $(\mathrm{n}=85)$ & 8 & 77 & 85 \\
\hline Diseased $(\mathrm{n}=75)$ & 19 & 56 & 75 \\
\hline \multicolumn{2}{|r|}{$\mathrm{p}$ value $=0.01$ statistically significant } \\
\hline
\end{tabular}


Table.3 HLA A1 positivity in B17 positive controls and diseased

\begin{tabular}{|c|c|c|c|}
\hline Study Group & A 1 Positive & A 1 Negative & Total \\
\hline Control $(n=8)$ & 2 & 6 & 8 \\
\hline Diseased $(n=19)$ & 15 & 4 & 19 \\
\hline \multicolumn{2}{|c|}{$p$ value $=0.0248$ statistically significant } \\
\hline
\end{tabular}

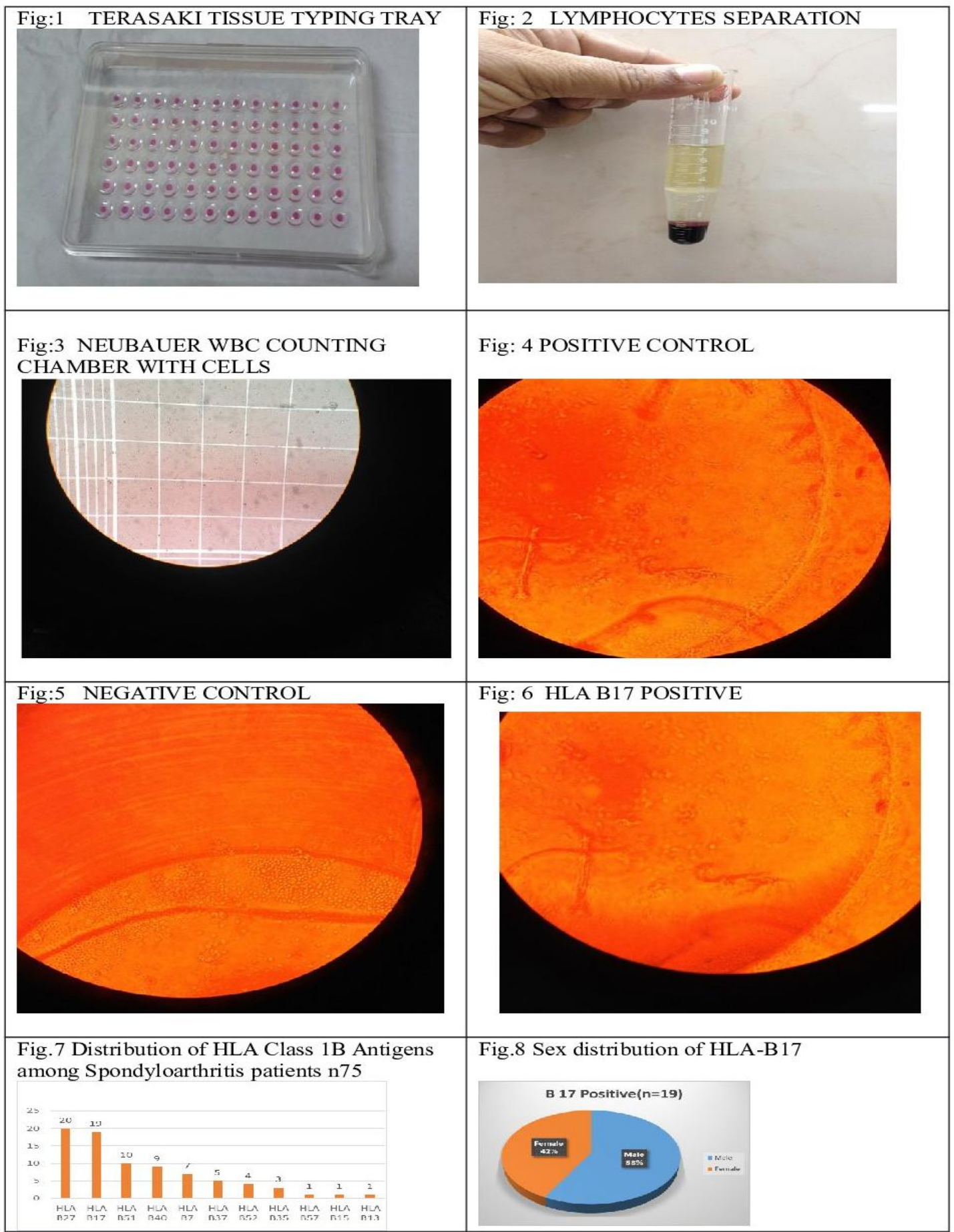


Population and family studies have shown that HLA-B60 increases susceptibility to AS. This applies to both HLA-B27 ${ }^{+}$and HLA -B27 persons. Contrary to the above studies we did not find any positive correlation with HLA-B60 and only one patient was B60 positive.

Up to 30 genes have been found to be associated with Spondyloarthritis. Psoriasis develops by the interaction of genetic, immunological, and environmental factors. Previous studies have reported a significant association of HLA class I and II antigens in patients with psoriasis which suggests the genetic background of psoriasis and role of HLA in its pathogenesis. Ray et al., had done HLA typing of patients with psoriatic arthritis in India and found a significant association with HLA - A1, B17. Psoriatic Arthritis is auto immune -mediated with defined HLA associations (B27, B17, DR4, DR7) and others. A study on Psoriatic arthritis has proved HLA-B27, -B17, -CW6, -DR4, -DR7 are associated with Psoriatic arthritis.

As per the common summary produced by Reliance Medical Information there was an increased prevalence of B17 in both juvenile and adult psoriatic arthritis. Although there was an increased prevalence of B17 in both juvenile and adult psoriatic arthritis, juvenile psoriatic arthritis showed an increased prevalence of A2, whereas adult psoriatic arthritis showed increased prevalence of B27, Bw39 and Cw6. These HLA associations differed from that reported in other arthritis. In our study, we found a significant association of HLA-B17 in SpA and HLA-A1 had an increased prevalence in B17 positive SpApatients (Hamilton et al., 1990).

Contrary to the above one patient with juvenile SpA was B27 positive and one was B17 positive in our study.
In conclusion, next to HLA-B27, B17 was detected as the common antigen in SpA. HLA-A1 had an increased prevalence in B17 positive SpA. The positive correlation between B17, A1 and SpA in our population shows a possible role of B17 and A1 in the pathogenesis of SpA and further researches are required to establish this. We recommend the complete HLA phenotyping by serological or other methods instead of looking for the presence of only HLA-B27.

\section{References}

Boyum, A. 1964. "Separation of white blood cells." Nature, 204: 793-794.

Braun, J., Sieper, J. Ankylosing spondylitis. Lancet, 369(9570).

Braun, J., van den Berg, R., Barallakos, X. 2010. update of the ASAS/ EULAR recommendations for the management of ankylosing spondylitis. Ann. Rheum. Dis., 70(6): 896-904.

Brown, M.A., Kennedy, I.G., MacGregor, A.J. 1997. Susceptibility to ankylosing spondylitis in twins; the role of genes, HLA, and the environment, Arthritis Rheum., 40(10): 1823-1828.

Dougados, M., Baeten, D. 2011. Spondyloarthritis. Lancet, 377(9783): 21272137.

Gary, S., Firestein, Ralph, C., Budd, Sherine, E., Gabriel, Iain, B., Mcinnes, James, R.O'Dell. Kelly's Textbook of Rheumatology Vol II, $9^{\text {th }}$ edition, Chapter 75, Page 1205.

Hamilton, M.L., Gladman, D.D., Shore, A., Laxer, K.M., Silverman, E.D. 1990. Juvenile Psoriatic Arthritis and HLA antigens. Ann. Rheum. Dis., 49(9): 694-7.

Kaipiainen-Seppanen, O., Aho, K., Heliovaara, M. 1997. Incidence and prevalence of ankylosing spondylitis in Finland, J. Rheumatol., 24: 496499. 
Khan, M.A., Khan, M.K. 1982. Diagnostic value of HLA-B27 testing in ankylosing spondylitis and Reiter's syndrome, Ann. Intern. Med., 96: 7076.

Khan, M.A., Kushner, I. 1984. Diagnosis of ankylosing spondylitis. In Cohen AS, editor: Progress in Clinical Rheumatology, Vol I, Orlando, 1984, Grune \& Stratton, pp 145-178.

Marc, C., Hochberg, Alan, J., Silman, Josef, S., Smolen, Michael, E., Weinblatt, Michael, H., Weisman. Rheumatology, volume $2,6^{\text {th }}$ edition, chapter 116 , page no 956 .

Poddubnyy, D., Rudwaleit, M., Haibel. 2011. Rates and predictors of radiographic sacroilitis progression over 2 years in patients with axial spondyloarthritis. Ann. Rheum. Dis., 70(8): 1369-1374.

Ray, S.C., Singh, T., Kaur, I., Suri, S., Sehgal, S., Kaur, S. 1990. Clinical profile of psoriatic arthropathy. Indian J. Dermatol. Venereol. Leprol., 56: 200-3. www.patient.co.UK/doctor/psoriatic arthritis pro
Rudwaleit, M., Jurik, A.G., Hermann, K.G. 2009. Defining active sacroilitis on magnetic resonance imaging (MRI) for classification of axial spondyloarthritis: a consensual approach by the ASAS/OMERACT MRI group. Ann. Rheum. Dis., 68(10): 1520-1527.

Terasaki, P.I., McClelland, J.D. 1964. Microdroplet assay of human serum cytokines, Nature, 204: 998-1000.

Terasaki, Pl. 1980. Ed., Histocompatilibilty testing, UCLA Tissue Typing Laboratory, Los Angeles CA.

Van den Berg, R., de Hooge, M., Rudwaleit, M. 2012. ASAS modification of the Berlin algorithm for diagnosing axial spondyloarthritis: results from the Spondyloarthritis Caught Early (SPACE)-cohort and from the Assessment of SpondyloArthritis International Society (ASAS)-cohort. Ann. Rheum. Dis.

Weber, U., Lambert, R.G., Pedersen, S.J. 2010. Assessment of structural lesions in sacrolliac joints enhances diagnostic utility of magnetic resonance imaging in early spondylarthritis. Arthritis Care Res., (Hoboken) 62(12):17631771.

\section{How to cite this article:}

Therese Mary Dhason, Euphrasia Latha Jairaj and Rajeswari Sankaralingam. 2016. Prevalence of Human Leucocyte Antigen B17 in Spondyloarthritis Patients in a Tertiary Care Center in South India Detected Microlymphocytotoxicity Test. Int.J.Curr.Microbiol.App.Sci. 5(7): 592598. doi: http://dx.doi.org/10.20546/ijcmas.2016.507.066 